

Scand J Work Environ Health 1990;16(2):85-95

https://doi.org/10.5271/sjweh.1812

Issue date: 01 Apr 1990

Biosafety considerations in industries with production methods based on the use of recombinant deoxyribonucleic acid.

by Andrup L, Nielsen BH, Kolvraa S

Affiliation: Danish National Institute of Occupational Health, Copenhagen.

This article in PubMed: www.ncbi.nlm.nih.gov/pubmed/2191426

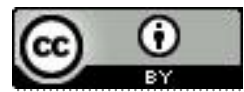




\title{
Biosafety considerations in industries with production methods based on the use of recombinant deoxyribonucleic acid
}

\author{
by Lars Andrup, MSc, ${ }^{1}$ Birgitte $\mathrm{H}$ Nielsen, DVM, ${ }^{1}$ Steen Kølvraa, MD ${ }^{2}$
}

\begin{abstract}
ANDRUP L, NIELSEN BH, KøLVRAA S. Biosafety considerations in industries with production methods based on the use of recombinant deoxyribonucleic acid. Scand J Work Environ Health 1990;16:85-95. Since no occupational accidents or diseases have been attributed specifically to the use of constructions containing recombinant deoxyribonucleic acid (rDNA), this paper evaluates the occupational health risks in industries utilizing genetically manipulated organisms mainly on the basis of theoretical considerations. Bacteria, filamentous fungi, yeasts, and mammalian cells in culture are in use. For each of these systems the possible hazards are considered. Concerning microbial production systems, infections are regarded as the main problem, but the risk of infection is considered extremely low. As for cells in culture, only dormant viruses are regarded as problematic, but well-defined production cell lines should not contain such undetected and dangerous viruses. Overall, the additional risks posed by rDNA-modified microorganisms are minor. Only long-term observations can, however, confirm this assumption, and consequently the highest feasible containment measures should still be used in the years to come.
\end{abstract}

Key terms: biological containment, biotechnology hazard, genetic engineering, risk assessment, risk management.

The last decade has seen a slow rise in the number of industries trying to base their production on the use of organisms modified by recombinant deoxyribonucleic acid (rDNA). Until now only a limited number of products synthesized in this way has been marketed, but the number has been limited mainly due to the costly and time-consuming research and documentation necessary before marketing is feasible $(1,2)$. From a theoretical point of view the number of companies that might be interested in this technique is very high. In Denmark, where a substantial portion of the industry involves the production and processing of food, pharmaceuticals, and chemicals, it has been estimated that companies representing as much as $20 \%$ of the gross national product will sooner or later use rDNA techniques (3).

In view of this potentially widespread use of rDNA methodologies in the workplace, it is relevant to consider the associated risks. Normally occupational risks are defined empirically, that is, through acquisition of knowledge about diseases and accidents that happen within a given industry. In this respect rDNA research and production appears to be rather unique, since no diseases or accidents have so far been described which

\footnotetext{
1 Danish National Institute of Occupational Health, Copenhagen, Denmark.

2 Institute for Human Genetics, University of Århus, Bartholinbygningen, Århus, Denmark.
}

Reprint requests to: Mr L Andrup, Danish National Institute of Occupational Health, Lersø Parkalle 105, DK-2100 Copenhagen $\varnothing$, Denmark. can be attributed solely to the fact that an rDNA construction was used (4).

In the absence of empirical data one is left with only theoretical speculations when trying to define areas where problems may occur and where more systematic research should be performed.

In this review we present our own views on these problems. Initially a brief description of the normal organization and function of a gene is given, followed by a brief description of the various elements needed to create an rDNA construction able to cause a cell to produce a given protein. Then some of the methods with which complex molecules are produced by use of rDNA constructions are reviewed, and in this context our views on potential risks in connection with the various kinds of productions are presented. Only the occupational health aspects are considered in this review, not the possible risks to the external environment. Finally, some consideration is given to risk management.

\section{ORGANIZATION AND FUNCTION OF THE NORMAL GENE}

\section{Chemical structure of deoxyribonucleic acid}

The information that codes for the numerous proteins produced in a cell is contained in the deoxyribonucleic acid (DNA). DNA is a double-stranded helical molecule. Each strand is built up by a sequence of nucleotides, and each nucleotide contains one phosphate and one deoxyribose molecule, which together form the backbone of the strand. In addition, each nucleotide contains a heterocyclic base. Four different bases are 
involved. In DNA these bases are adenine, cytosine, guanine, and thymine (5). The code is contained in the sequence of appearance of these four bases in the strand so that three bases in a given sequence specify one amino acid in the protein which the gene codes for. The overall base sequence thus gives both the identity of the amino acids and the sequence in which they will be positioned in a growing polypeptide chain. The fundamental basis for rDNA productions is the fact that the genetic code is universal and therefore allows the genes from different organisms to be read similarly, the result being the production of the same polypeptide chain even if the gene and the synthesis apparatus are from two different organisms.

\section{Transcription and translation}

When the information encoded in the DNA is needed for the production of a polypeptide chain, the code is copied onto a so-called mRNA molecule ( $\mathrm{m}$ stands for messenger and RNA for ribonucleic acid). The mechanism by which the code is copied involves positioning of specific bases opposite the bases in one of the strands of the double-stranded DNA, a complementary copy of the DNA code thereby being obtained. This process is called transcription. In its simplest form mRNA is then transported to the ribosomes, and polypeptide chains with the sequence encoded in the mRNA are synthesized. This process is called translation. In bacteria, which have no nucleus, the transcription and translation can take place simultaneously.

\section{Regulatory sequences}

Several problems arise when attempts are made to use the simple design of introducing a human gene into a bacterial host in order to acilicve the synthesis of a desired protein in the bacteria. Intense research over the last decade has demonstrated that several regulating sequences exist both closely in front of the gene and, for eukaryotic cells, far away from the gene (6). Such sequences determine both the degree of expression (meaning the amount of protein being synthesized) under various conditions and the type of cell in which expression will occur (species and/or organ specificity).

\section{Splicing}

Furthermore, a process called RNA-splicing occurs in higher organisms but not in prokaryotes. Splicing is a process by which mRNA is modified after transcription but before leaving the nucleus. This process is necessary since eukaryotic genes are often interrupted by noncoding sequences inserted at various locations in the gene. The noncoding sequences are termed introns, and the coding sections in between are called exons. Splicing involves the removal of noncoding sections of the mRNA and, thereafter, end-to-end ligation. This process results in a shortening of the mRNA. The fact that prokaryotes do not have this system signifies that such an organism will try to translate the noncoding information of the eukaryotic mRNA also and thereby produce erroneous polypeptides. The molecular mechanisms behind splicing are slowly being characterized (7).

\section{Posttranslational modification}

A number of processes under the common name of posttranslational modification are involved in the construction of complex molecules in eukaryotic cells. These modifications may comprise a specific folding of the newly synthesized polypeptide chain, assembly of several polypeptides to form the final protein molecule, and perhaps coupling of various compounds to the polypeptide chain. The compounds in question are phosphates, sugars, vitamin metabolites, etc (1). These final modifications often require the participation of other enzymes, enzymes that are coded for by other genes. Therefore it is not certain whether or not a proor eukaryotic host cell that is able to synthesize the primary polypeptide chain has the enzyme battery necessary to make the final protein.

\section{THE rDNA CONSTRUCTION}

In order to make a DNA construction for use in rDNA production, certain procedures to isolate the different necessary elements are mandatory. The DNA elements necessary for an operative construction are the gene (preferably without the introns), certain regulating sequences (ensuring high and stable expression of the gene in the host organism), and a vector (which can be regarded as the carrier of the construction). The tools used in rDNA technology to create such a construction have not been described in this report. (For a review see reference 8 .) Instead a few comments on each of the elements in the construction are offered.

\section{The insert}

With regard to the isolation of specific genes from the DNA of higher animals, several techniques exist which, as a starting point, use existing knowledge about the protein in question. It may be in the form of antibodies against the protein or in the form of data about the amino acid sequence. (For a review see references 9 and 10.) With the use of this knowledge it is possible to retrieve the desired gene. 


\section{Regulatory sequences}

The regulatory sequences needed to give a strong expression of a gene depend very much on the host in use. In general, the strongest regulatory sequences are derived from various viruses, but often the host specificity of the virus puts restraint on the choice of the host cell. For many microorganisms too little is known about the regulation of gene expression to construct the ideal vector.

\section{Vectors}

Common for most vectors is the fact that they are small, naturally occurring DNA elements that can be taken up in various bacteria and cells; they multiply together with the chromosomal DNA of the cell. For bacteria, the vectors most commonly used are those based on plasmids or phages. The plasmids are small circular pieces of DNA which are naturally occurring elements of many bacteria. They are present in a varying number of copies, depending on both the plasmid and the host, and code for a number of functions, including various antibiotic resistances (11). The phages are bacteria-specific viruses which can enter the cell, multiply with the cell or, after proliferation, lyse the cell in order to infect other cells. With the use of both plasmids and phages several rDNA vectors have been developed. They are more or less artificial constructions containing the sequences necessary for basic functions. Some of the vectors are designed to cause the expression of genes ligated into the vectors (expression vectors), and others have a more or less unaffected ability to infect cells by natural means. For cells from higher animals, the vectors used are all based on viruses which normally infect these cells $(8,12)$. Again, these vectors are often modified in many ways, eg, made defective so that they do not normally cause lysis and death of the cell and thereby give the system more stability.

\section{RISK IDENTIFICATION AND EVALUATION IN PRODUCTIONS USING IDNA TECHNOLOGY}

\section{Range of products}

The major impact of rDNA technology has, until now, been in the production of endogenous and exogenous proteins for therapeutic and diagnostic use. Most of these proteins fall in one of the following five categories: hormones (eg, human insulin, human growth hormone, calcitonin, somatostatin, relaxin, parahormone, erythropoietin), immunomodulators (eg, interferones, interleukines, colony-stimulating factors), blood products (eg, tissue plasminogen activator, factor VIII, factor IX, human serum albumin, alpha-1antitrypsin), antitumor agents (tumor necrosis factor, immunotoxins), and vaccines (hepatitis B) $(1,13-15)$.
Future developments, apart from an increase in the number of protein products, will probably show a spread of rDNA technology to many other areas, such as the synthesis of nonprotein compounds through the construction of whole pathways in living organisms (16), the production of nonprotein biopolymers (17), and the catalysis of specific reactions such as bioleaching in mineral processing (18). These applications are, however, very much at the experimental stage at the moment and have not been considered in this review; instead it is systems for the industrial production of peptides and proteins that have been included.

\section{Production systems}

The biological systems with which to produce rDNAcoded peptides and proteins are either microbial (bacteria, filamentous fungi, or yeast) or based on mammalian cells in culture. In addition, the possibilities of producing complex molecules in transgenic animals are currently being investigated.

These systems have been described in the following discussion, together with considerations concerning the possible risks.

\section{Microbial systems}

Bacterial systems. The simplest system and the system which is still the most widely used in the rDNA production of proteins is based on the incorporation of the coding DNA, without introns, together with regulatory sequences, into a suitable bacterial vector, which is then propagated in a bacterial host. The advantage with bacterial systems lies in the simple substrate requirements of bacteria, their ability to grow almost unrestrictedly in huge fermentor vessels, and, at least for some products, the possibility to design the system in such a way that the host secretes the polypeptide into the medium, downstream processing therefore being made easier (2). The disadvantages are mainly concerned with the rather primitive processing systems of the prokaryotes in that these systems result in a lack of splicing [can be overcome by the use of copy DNA (cDNA) sequences in the insert] and a lack of more refined posttranslational modifications. Therefore, in general, these systems are used in the production of more simple proteins (1). The possibility of producing precursor polypeptide molecules, which can later be modified in other systems, are at present being investigated.

The most popular host organism is still Escherichia coli. This bacterium is by far the best characterized microorganism. Many strains have been isolated, including laboratory strains that are mutated to such a degree that they are not able to survive outside controlled culture conditions.

Of the bacterial hosts, $E$ coli has, however, one disadvantage, namely, the tendency of forming inclusions (2). Inclusions are insoluble aggregates of protein and 
DNA which form intracellularly when foreign genes are overexpressed in $E$ coli. This phenomenon most likely occurs because of a limited ability to secrete proteins. It is possible to stimulate secretion by the addition of signal sequences to the polypeptide chain, but this step only transfers the protein as far as the periplasmic space, where accumulation and protein degradation then occurs. Another disadvantage is the production of endotoxins from the outer cell wall of $E$ coli during degradation. Both of these problems can be avoided with the use of members of the Bacillus genus (19). These bacteria are genetically and biochemically less characterized than $E$ coli. Most strains are nonpathogenic, some are nonsporogenic, and they grow aerobically. The greatest advantage is, however, that they, being gram-positive, have a much simpler cell wall than $E$ coli. Therefore they secrete overproduced proteins much more easily and do not form endotoxins.

A number of other bacteria (eg, various Lactobacillus and Streptomyces species) are now being tried as hosts for production, but the experience with these is much more limited, and they will not be considered in this report.

In practice the fermentations using both unmodified and rDNA-modified microorganisms involve initially filling the fermentor with medium, sterilization, and cooling. Then seeding is performed by the addition of the production organism, and the fermentation is run with stirring, aeration, and a supply of extra nutrients. When the fermentation has reached a certain level, it is either terminated (batch production) or the fermentation mixture is continuously removed and replaced with new medium (continuous production). The produced compound is rescued by an initial separation of the medium and cells followed by the extraction and isolation of the product from either the filtrate or the cell residue. These and the following steps are called the downstream processing.

Filamentous fungi. Filamentous fungi are of considerable industrial importance since they produce about $60 \%$ of known antibiotics. They are eukaryotes, grow in filamentous form, and produce spores. They do perform some posttranslational modifications but are, apart from this, comparable with bacteria. A few rDNA products are currently being produced in fungi (13), and an increase is to be expected.

Yeast. Yeast is also a eukaryote, possessing the ability to perform posttranslational modification. The modifications, eg, glycosylation, are, however, not always exactly similar to what is done in mammalian cells. Yeast is therefore an intermediary type of host, being a eukaryote able to be cultivated like bacteria on defined, simple media. In addition the generation time is much shorter than for other eukaryotic cells. As an industrial production organism, yeast has a very long history, especially for alcohol fermentation and baking. One of the areas where rDNA manipulation on yeast has been tried is therefore not surprisingly in the brewery industry (20). Attempts have aimed at reducing the use of raw materials, improving the quality of beer, and developing new beverage products. In addition yeast is being tried as a production organism for various other products, eg, vaccines.

Possible risks in the use of microbial systems. The inherent risks of working with microbial production systems can be related to the following: (i) the microbiological agent involved in the fermentation (the host), (ii) the biological material, which is either present in the microorganism (eg, the rDNA construction) or produced by the organism (eg, toxins), (iii) the final product, which the production is aimed at, and (iv) various chemicals and other substances involved.

In order to have a harmful effect, the microbiological agent has to gain access to the worker. In general, the following events have to occur before this event takes place: (i) the microorganism has to escape to the work environment, (ii) the microorganism has to spread and multiply, and (iii) the microorganism has to establish itself on or in the worker.

Living microorganisms can escape during production either from the fermentor vessel during the fermentation or, in case the content of the vessel cannot be sterilized prior to downstream processing, during the recovery of the product. The risk of an accidental escape from the fermentor vessel can be minimized by proper design, and therefore massive contamination from bioreactors is almost always due to operator failures. The risks for accidental escape are somewhat greater during downstream processing since it involves several procedures, such as centrifugation, for which full physical containment is notoriously difficult to maintain and for which aerosol formation is a problem.

Both the design of the system and the operational procedures should obviously be optimized for the highest degree of containment so that massive escapes can be avoided. Absolute physical containment is, however, extremely expensive, and therefore minor leaks are probably rather common in most situations.

The extent to which an escaped microorganism will spread and multiply outside the vessel is generally difficult to evaluate since this, among other elements, involves assessments of interactions between the rDNA organism and the microorganisms in the existing ecosystems. Such assessments have turned out to be difficult to perform.

Some knowledge is available concerning spread in terms of the environmental transport of microorganisms. Several studies have investigated the airborne spread of microorganisms in aerosols both indoors and outdoors. These studies revealed that especially $E$ co$l i$ is very susceptible to drying in aerosols and will, when measured as colony-forming units after deliberate release in aerosols, disappear within a few minutes (21, 
22). In contrast the organism can be found in moist dust and similar environments for weeks. Other organisms, more resistent to drying (eg, due to sporulation), can be carried for hundreds of kilometers and still be viable $(23,24)$. Therefore, once a microorganism is released in aerosol in a building, it can in theory spread as a viable organism to all locations in the building and to the exterior.

Since, however, most accidental escapes are in the form of minor leaks with only a limited number of microorganisms escaping, the consequences will only be serious if the microorganism finds ways to multiply in the environment. Then so-called biological containment becomes relevant.

Biological containment is present if an rDNA organism possesses a number of characteristics making it unable to, or not very good at, multiplying outside the vessel (25). Many biological containment features are added in the form of very specific growth requirements, which cannot be met outside the vessel. In addition mutations, making a microorganism in other ways weak when compared with wild types of bacteria, fall into this category. Various suicide systems are also being developed; with these systems an rDNAmodified microorganism contains a gene coding for a lethal toxin which is activated either if specific culture conditions are not present or at random after a certain period of time $(26,27)$. These systems have not been tried in production yet, but they seem promising. They are, however, sensitive to accidental mutations that inactivate the toxin gene.

A phenomenon that clearly weakens the biosafety of organisms with a high degree of biological containment is the fact that many of the characteristics giving biological containment are derived from the host organism. This is a problem since it is to a great extent the rDNA construction and the information encoded that must be worried about. A way to cross-circuit the safety measures of biological containment would therefore be for the rDNA construction to escape from the weakened primary host to a more robust wild type of bacteria (28). This process of escape to other bacteria is called horizontal transfer. Such transfer can occur through three different processes, depending on the relationship between the bacteria.

First, naked DNA, released, eg, through the death and lysis of the donor bacterium, can be taken up through the cell wall of the recipient organism by the process of transformation.

Another process for horizontal transfer is so-called transduction, which means transfer via a bacteriophage which accidentally carries a piece of rDNA from one cell to another.

The third process is conjugation. This is the "natural" process by which one bacterium, using a specialized organ (sex pilus), can transfer plasmids and in some cases chromosomal DNA to another bacterium. This phenomenon is common and results in a constant reshuffling of the total pool of DNA within a wide range of microorganisms. The phenomenon is so widespread that it has been suggested that microorganisms should not be considered discrete organisms or true species in the sense that eukaryotes are. Rather, the microbial community resembles a vast computerized communication network, a global organism, whose parts share most genetic information (29). Accepting such a notion means of course that an escaped rDNA construction may in time be spread through the whole bacterial community.

The likelihood of spread through the various mechanisms can be minimized through the proper design of the vectors and the correct choice of host bacteria, but no one will probably claim that horizontal transfer can ever be totally controlled through the design of the system.

The probability of the spread and multiplication of an escaped rDNA construction is, however, considered minor due to the fact that several specific events have to occur before the rDNA construction can survive outside the vessel.

A microorganism which has escaped and multiplies in the work environment may establish itself in or on the workers exposed to it. Several factors are important in such cases. First, various intrinsic characteristics of the escaped microorganism are of course relevant. Second, factors such as size of dose and site of invasion are important. This importance can be illustrated by the doses of microorganisms needed to cause infection. The necessary dose for humans varies considerably among the various pathogenic microorganisms. Very few organisms are needed for infections with some viruses, especially viruses which gain access through the upper respiratory tract (measles, influenza, polio, etc). Similarly, the number of microorganisms that can cause disease by intradermal inoculation in diseases such as scrub typhus and syphilis are very low. The opposite is generally the case with microorganisms that cause gastrointestinal diseases, for which the route of entrance is ingestion. In such cases many organisms $\left(10^{5}-10^{9}\right)$ are needed, most probably due, first, to excessive killing in the acidic stomach juice and, second, to the need for a sizable initial inoculation in the gut in order to compete efficiently with the natural flora in the first phase of the infection (30).

Fortunately most of the microorganisms used currently in rDNA-based production are organisms that invade though ingestion, and it is therefore considered unlikely that minor leaks would result in ingestion of the necessary number of organisms. Probably only two situations could pose a problem. One would be when specific circumstances confer advantage to a recombinant microorganism, eg, in the case of a worker receiving the same antibiotic as the rDNA construction confers resistance to. The other would occur when an organism has a natural ecological niche on the body of the worker to "return to." If it can survive there without disadvantage, it could cause disease, eg, by producing a toxin coded by the rDNA construction. 
An example would be a normally occurring enterobacterium, which reestablished itself in the gut after receiving an rDNA construction coding for a toxin. The often heard argument against such a mechanism is that an enterobacterium containing an expressed rDNA construction will have a disadvantage due to this "extra luggage"' and therefore will be eliminated by its wild type of ancestors. Recently, however, observations have been presented that seem to indicate that a foreign plasmid can, after some time, form a symbiotic relationship with the new host and then is conferred with selective advantage instead (31).

Adverse effects caused by a microorganism which has established itself on a human being can, in general, be either in the form of infection or an allergic reaction. In order to cause an infection, the microorganism has to have pathogenic capabilities. Only very few of the many different microorganisms have this ability (32). This scarcity is not surprising since efficient pathogenicity is due to the balanced actions of several genes. These genes code for functions such as ability to attach to the surface of the organism to be infected, mechanisms for obtaining entrance, mechanisms for interference with the phagocytotic processes, toxin production, etc.

Three ways seem open when mechanisms by which an escaped microorganism can be pathogenic are considered. First, the host bacterium can deliberately have been chosen to be a pathogen. This is seldom the case, but it does occur in connection with the production of vaccines and similar products. Then extra tight physical encapsulation is obviously needed. This precaution makes such productions extremely expensive, but technically full containment can be achieved, as has been shown by the longstanding manufacturing of vaccines from even the most dangerous pathogens. Second, the host bacterium may start out as a wellcharacterized, apathogenic organism, but during the fermentation natural mutations result in the creation of a new genotype with pathogenic abilities. Given the thousands of generations of continuous fermentations and the natural mutation rate of bacteria, substantial genetic changes seem likely. The probability of a change to pathogenicity seems, however, indefinitely small due to the fact that several distinct genes are needed for pathogenicity, combined with the fact that pathogenicity confers no advantage upon an organism grown in a fermentor vessel. Third, the host may be apathogenic, but the rDNA construction confers pathogenic ability. This will be a rare situation since a well-defined rDNA construction presumably confers only one ability, such as toxin production, to the host. Therefore other abilities, just as necessary for the development of disease, will still be lacking.

The overall conclusion is therefore that exposure to microorganisms with pathogenic capabilities will only occur in some defined circumstances. The likelihood of an accidental development of pathogenic capabili- ties outside these specific circumstances is extremely low and can be ignored.

With regard to allergy, workers can, in theory, become sensitized to any microorganism with which they come in contact. The general experience is, however, that allergic reactions of patients towards intact bacteria established in or on the patients are rare.

The risks of exposure to the rDNA construction as such, or to endo-and exotoxins, is greatest during the downstream processes, especially in productions in which the product is located intracellularly and in which disruption of the cells is necessary. Exposure may, however, also occur if the product is excreted simply because a certain cell death and lysis occurs during the fermentation.

Risks associated with the recombinant vector itself are probably minimal. This situation is due to the fact that, although both plasmids and phages must be considered infectious agents, their host spectrum includes only prokaryotes. In addition, the spread of their genes is a normal event that occurs constantly in nature (mediated by conjugation or transduction). Man has therefore always been exposed to these sequences, although the vectors used in rDNA work are artificial constructions with sequences from many sources.

Endo- and exotoxins are known to be able to cause both toxic and allergic reactions. Their escape will, however, probably be limited to specific operations, and this circumstance, combined with the fact that sensitive test methods exist, means that proper measures for protection can be taken (33).

The risks of accidental contact with the gene product will also be greatest during the downstream processes. The gene products are, however, as are the toxins, chemical compounds and not living organisms. Potential risks can therefore be assessed according to conventional toxicologic principles.

The various chemicals involved in production with rDNA biotechnology do not differ from those used in other productions, and the associated hazards and risks have not been considered in this report.

\section{Mammalian cells in culture}

Growing mammalian cells in cultures is at present the only commercial method for producing those recombinant proteins which need specific conformations or other kinds of posttranslational modifications that cannot be achieved in microbial fermentation systems.

Cells in culture can grow either as anchorage-dependent or ançhorage-independent cells. Most cells from blood, including the hybridoma cells used to produce monoclonal antibodies, grow anchorage independently. Tissue cells almost always grow anchorage dependently, and, apart from the production of monoclonal antibodies, it is the general experience that the best results with rDNA production are achieved in cells which are anchorage-dependent (13). 
The advantage gained with the use of production in cell cultures is that the final product is obtained even when very complex proteins are being produced. Until now, production in mammalian cells has, however, been limited due to the fact that these cells grow very slowly, are larger and more fragile, and have more complex nutritional requirements than microorganisms. In addition, they are far more susceptible to contamination. Until recently these disadvantages were considered prohibitive for more widespread use, but research in recent years has indicated that with proper measures cell cultures can be established for most cell types (34). In addition many kinds of cells can be made to grow to almost as high densities as microorganisms if protected efficiently against physical damage (13).

Several products are presently being produced in non-rDNA-modified cell cultures. They include various virus vaccines, immunoregulators, hormones, enzymes, and tumor-specific antigens (14). On the borderline of rDNA-modified cell lines are hybridoma cells, which are used to produce a vast number of monoclonal antibodies, both for diagnostic and therapeutic purposes (35). Production in cell cultures that are rDNA-modified in the classical sense is still mostly at the experimental stage. One promising field is the development of viral vaccines through the expression of viral subunit proteins by such genetically modified cell lines. Other products, which are presently being developed and tested, are growth hormone, tissue plasminogen activator, factor VII, and factor VIII (14).

Possible risks in the use of mammalian cell cultures. At first glance, cells in culture would seem far less problematic than microorganisms due to the fact that the cells are extremely "biologically contained" in themselves because of the very stringent nutritional requirements. It thus seems very unlikely that any cells that escaped from a culture vessel would survive for any appreciable period of time, and it is just as unlikely that they would find an environment in which they could establish themselves in continuous growth.

Any hypothetical risks in connection with cell culture production would therefore probably occur in connection with any release of an active agent or compound from the cells. These hypothetical agents or compounds can, in principle, be either of exogen or endogen origin. In this context exogen origin means agents and compounds that are not normally present in the cell culture, but which are present in the production cells either by accident or on purpose. Exogen agents, which are unintentionally present in the cultures, are typically microorganisms that have infected the cells, eg, viruses and mycoplasmas. Although these agents can cause disease in the worker if they escape, they will cause far more trouble for the production as such, and a producer will normally use several test systems in order to detect and avoid the occurrence of such infections in the cultures.
Foreign compounds which are present in the cells on purpose include the rDNA construction as such, the product which the inserted gene is coding for, and any viral elements which have been integrated into the high molecular DNA in order to make the cells immortal (36).

With regard to the rDNA construction, then, the vector is the most probable source of problems. As previously mentioned, expression vectors for human cells are based on viruses, ie, agents that can cause infections in man. The risk for such infections is, however, minor due to specific circumstances in the construction of virus vectors. The construction is based on the identification of a region of the virus genome, which is not essential for the normal life cycle, and subsequent replacement of this region with the foreign DNA. Since only a limited part of the viral DNA is unnecessary and a virus particle can contain only a certain amount of DNA in order to be packed normally, there are severe constraints on the amount of foreign DNA such a virus can contain. It is possible to clone larger fragments of DNA, using a viral vector, but in such cases essential genes have to be deleted and the vector virus is then defective and cannot go through the life cycle alone (12). In such cases propagation of the recombinant virus can only be achieved by the presence of a helper virus. Then the protein elements, which are absent in the cell due to the deletion of their genes from the vector viral genome, are supplied from the genome of the helper virus, and a normal life cycle is achieved as long as the helper virus is present.

From the standpoint of safety it is generally considered an advantage that most virus vectors are defective, since then the properties necessary for infectivity are normally absent. It is, however, necessary to be aware of the fact that coinfection with a helper virus in addition to the virus vector removes this safety.

The risks due to the product can be assessed by traditional toxicologic methods, and they have not been considered in this review.

Another issue that has resulted in a great deal of controversy with respect to continuous cell lines is the possible presence (on purpose or accidentally) of sequences which confer immortal growth capabilities to the cells. Industry often prefers these cell lines because of their stability. As a consequence of their immortal growth capability these cells can, however, form tumors when inoculated into immunosuppressed rodents (37). There has been a longstanding argument concerning the possibility that the tumorigenic ability of continuous cell lines might be conveyed to persons in contact with the cells. Such tumorigenicity might theoretically be mediated through several mechanisms. First, accidental inoculation of the immortalized cells might result in tumor formation. A major argument against this mechanism is that such cells will be immunologically rejected by the recipient organism, in agreement with the fact that such cells are only tumorigenic in immunosuppressed animals. In all likelihood, a tumor 
would only occur if the compatibility of the human leucocytlocus $\mathrm{A}$ is total (in practice meaning that the transformed cell line should originate from the person who is accidentally inoculated).

Another possible way for transformed cell lines to cause tumor formation in persons handling them would be by contact with cellular proteins with transforming power. Transforming genes exert their effect through proteins $(38,39)$, but such a risk would only be realistic if the protein in question should transform through action on the cell surface (which some of the oncoproteins actually do) (39). In addition, the exposure should be constant, since such proteins act through reversible activation of cell surface receptors. Obviously, without the corresponding DNA to create continuous production of the oncoprotein, continuous exposure will probably never occur.

A third possibility for the transfer of tumorigenicity from continuous cell lines would be through exposure to cellular DNA. Experiments with injections of as much as $500 \mu \mathrm{g}$ of the total DNA from cells with an activated oncogene did not give rise to tumor formation in newborn rats and guinea pigs. Neither did injections of similar amounts of cloned oncogene (40). It thus seems that the integration and expression of oncogenes from inoculated cellular DNA does not occur in practice.

A special problem is the possibility of total or partial viral genomes integrated in the DNA of cell lines. There is always the possibility that culture conditions can induce the expression of such dormant viral genomes and result in the production of infective virus particles. The risks posed by such viruses are in all likelihood small since most of these viruses will belong to strains that humans have been exposed to constantly and which therefore do not pose a new hazard. In addition they have, in the case of nonhuman cell lines, no host specificity towards humans. It must, however, in this context be borne in mind that the cooperation of a helper virus can alter the host specificity barriers.

All in all, the probability of acquiring a serious infection from dormant virus genomes in cell cuitures is considered extremely low. This conclusion is in agreement with the fact that many transformed cell lines have been grown in research laboratories for decades without any reported problems for the persons working with them.

\section{Transgenic animals}

By simple logic the best way to circumvent the problems caused by the delicacy of cells in culture would be to let them stay in the animal, in which case the whole animal would be the bioreactor. Then the rDNA modification is done through the process of injecting the rDNA construction (containing coding sequences and regulatory sequences) into the pronucleus of a fertilized ovum, reimplanting it into a foster mother, and letting the resulting transgenic animal be born. The idea is then that the rDNA construction is integrated into the DNA of the stem cells and ultimately is present and expressed in all the body cells of the animal.

This strategy has been tried extensively during the last several years, first with mouse single-cell ova (41) and later on with a number of other animals (rabbits, sheep, pigs, and cattle) (42). The first gene to give successful expression was the rat growth hormone gene which was injected together with the mouse metallothionein promotor into mouse ova (41). Several offspring from these series exhibited very high levels of growth hormone in serum and grew to two to three times the normal size. Later, ova of higher animals have been injected with similar constructions containing growth hormone, but the success rate has been noticeably lower than with mice. In addition the few animals that did get (marginally) increased levels of growth hormone did not grow to larger size but instead exhibited some side effects such as diminished resistance to infections, increased mortality, and infertility (42).

After these initial disappointments concerning the possible use of larger animals as bioreactors, much of the research has turned to attempts to create transgenic animals with the expression of foreign proteins only in single organs. The organ that has been experimented with the most intensively is the mammary gland. The logic behind this strategy is that less adverse systemic effects are expected if the rDNA product is expressed only in an exocrine organ, and furthermore purification from milk is expected to be without complications. Several experiments have been done, most using the incorporation of the gene in question into the betalactoglobulin gene, the result being expression only in the mammary gland. The experiments have been performed mostly on sheep, and expression in milk has been achieved for factor IX and alpha-1-antitrypsin, although only in minor amounts (42).

In summary it can be concluded that the use of transgenic animals as bioreactors seems promising, but it is far too early to say if it will be a feasible alternative to cells in culture.

It is difficult to see any major risks associated with the handling of transgenic domestic animals. As has been stated, the risks are substantially lower when the escaping rDNA-modified organism can be "called back." The arguments against the use of transgenic domestic animals have therefore mostly dealt with the ethical issues of using (or misusing) animals in this way.

\section{RISK MANAGEMENT IN rDNA-BASED PRODUCTION}

Increased biosafety in modern biotechnology must be achieved through the minimization of both the likelihood and the severity of the consequences of unwanted events. The processes by which to achieve this goal can be divided into the following three elements: risk iden- 
tification, risk estimation, and risk management. The first two elements have to do with the identification of risks and the estimation of the probability for such risks to occur, as well as attempts to predict the overall consequences of such events. By contrast, risk management has to do with actions to be taken.

The earlier sections of this review have concentrated mainly on risk identification. It has, however, been hampered by the fact that no adverse effects of rDNAbased biotechnology have thus far been published. We have therefore been forced to rely on the construction of various scenarios which, at least in theory, might pose a hazard and then try to estimate the probability of their occurrence. Such a strategy has obvious drawbacks due to the fact that basic scientific knowledge about such aspects as horizontal transfer and the stability of ecosystems is limited.

Therefore, in spite of the fact that available evidence might suggest that no rDNA-modified microorganism has caused health problems to human beings so far and that theoretical considerations also point to risks being very minor, it must be stressed that ultimately only epidemiologic studies over $20-30$ years can confirm the assumption that there is no special risk for workers in rDNA production.

It is therefore not surprising that both competent researchers and politicians have hesitated to conclude definitely that there is no danger. Instead, the present opinion of many experts is that optimal care should always be taken to ensure that the production organism, the production equipment, and the operational procedures are all designed in such a way that biosafety is optimal (25).

\section{Containment}

Two different types of containment have been defined, namely, physical and biological containment (25).

\section{Physical containment}

The concept of physical containment refers to all measures that aim at reducing the exposure of workers, other persons, and the outside environment to potentially hazardous microorganisms. Physical containment is usually considered under two headings, namely, primary and secondary forms.

Primary physical containment refers to devices that separate the operator of a given process from the agent. These are the relevant measures when occupational health is considered. Precautions then focus on the design of fermentor vessels, including ways of sterilizing fluids escaped during sampling, and exhaust air released on purpose during fermentations. Efficient encapsulation of the downstream processes until a point where no risks are present is also a part of these measures.

It is important to stress that full containment is not obtained by physical containment devices alone. Just as necessary is scrupulous adherence to operational practices. Several recommendations have been published in this respect. For reference see the recommendations of the Organization for Economic Cooperation and Development (OECD) (25), in which good industrial large-scale practices are defined. Instruction and education of the work force in operational practices is therefore necessary before full benefit can be obtained from physical containment systems.

Secondary physical containment is concerned with the protection of the environment beyond the confines of the production plant. Relevant measures are building design, ventilation design, waste disposal, etc. These aspects have not been dealt with in this review.

In the control of the physical containment of fermentor equipment, it is important to be able to monitor the surroundings for the presence of the utilized microorganism, especially when pathogens or potentially harmful microorganisms are being worked with. Several methods for the detection of viable microorganisms have been published. The most commonly used methods involve sampling the airborne microorganisms either according to the principle of impaction or on membrane filters (43). The detection is based on the viability of the microorganisms, and therefore factors such as the choice of growth media, the collection time, and the storage of the sample are important if excessive killing of the organism, and thereby also unreliable results, is to be avoided. Genetically engineered microorganisms are often weaker than wild types and are therefore often difficult to detect. Generally speaking, microorganisms in the air are associated with a complex of organic material, fibers, dust particles, and other microorganisms. Especially other microorganisms are a problem when the sampling procedures are based on microbial viability since these wild types of microorganisms tend to overgrow the less viable production organism. Therefore more untraditional methods must be considered. Such methods could be based on the demonstration of gene products in the work environment or the detection of DNA sequences specific for the rDNA organism, eg, by the polymerase chain reaction (44).

\section{Biological containment}

The concept of biological containment has been discussed in connection with the likelihood of an escaped microorganism multiplying in new environments. In this review it will only be emphasized that one of the basic principles of the OECD recommendations (25) is always to construct the biological system with as much biological containment as possible, given the needs of the process.

\section{Medical surveillance}

Another aspect of risk management, which has given rise to some controversy, is the concept of medical sur- 
veillance of workers. The purpose of medical surveillance is to find, as early as possible, those persons in the work force who, for some reason, may have higher risk of acquiring an occupational disorder.

Medical surveillance is usually performed through the evaluation of the health status of the workers before, during, and after a work period. Several strategies can be used in various combinations.

Considering that the main health problems in rDNAbased production in all likelihood are infections and allergies, one strategy is based on the idea of identifying, as early as possible, those persons likely to run into these problems. Therefore the whole force must be screened for individuals with diminished resistance to infections (immunosuppression, severe general disease, antibiotic treatment, widespread defects in skin or mucosal membranes, etc), and also persons with atopic predisposition must be screened for.

Another strategy is to look for the actual occurrence of diseases that might be caused by the production organism. This procedure includes penetrating diagnostic procedures in order to get a specific diagnosis as soon as an employee has an, even shortlasting, period of illness. Intermittent blood sampling followed by storage of serum for continuous serological monitoring for contact with specific microorganisms is another useful procedure.

A third and still very controversial strategy is to screen for a genetic predisposition towards occupational disorders (45). There is no doubt that the development of methods for detecting variations on the DNA level makes it technically possible to identify individuals with genetic predisposition, but a sensible development in this area has, to some extent, been sabotaged by examples of clear misuse of such techniques that have resulted in discrimination against single persons or ethnic groups. For a discussion of the ethical issues see reference 46.

A detailed description of arguments for and against medical surveillance in occupational health will not be given in this review, but most arguments against have focused either on the risks for discrimination against persons with a certain degree of predisposition or on the question of whether extensive medical surveillance, followed by the exclusion of workers at risk, would only delay the establishment of technical preventive measures in production.

As has already been stated, one of the major limitations when risk estimations are performed in the field of biotechnology is the lack of precise knowledge on which to base the estimates. Knowledge is lacking both concerning the basic biological mechanisms that might lead to problems and the types and frequencies of specific health problems in these industries.

While basic biological mechanisms will be characterized in detail in due time independently of occupational health aspects, increased knowledge of the health problems involved requires more systematic epidemiologic studies. It must, however, be stressed that such studies are slow at giving specific answers. It must therefore be relevant, in these studies, to include medical surveillance programs aimed at detecting mild or subclinical infections caused by the production organism or parts of it in order to accelerate such studies. This aspect is of course another argument for at least some kind of medical surveillance program in industries using rDNA-based production.

\section{Regulations}

Since the techniques of genetic manipulation emerged in the early 1970s, several guidelines for working with rDNA organisms have been published. First the National Institutes of Health (NIH) in the United States formulated guidelines for research involving rDNA molecules. They were published in 1976. At that time there was considerable public concern over the hazards posed by this new technology, both to workers and to the community. The last decade has shown that many of these concerns were unfounded, and the NIH guidelines have been loosened several times. Now work with $E$ coli KI2, Saccharomyces cerevisiae (bakers' yeast), and Bacillus subtilis is regarded as safe, provided that the cloned DNA does not contain any "harmful" genes and that no self-transmittable vectors are used.

Most countries have chosen to observe the NIH guidelines or make their own versions. As previously mentioned, in 1986, the OECD published its recommendations (25); they are the work of an ad hoc group of government experts established in 1983. This was an important step towards international standardization.

Denmark has enacted specific legislation concerning work with rDNA organisms. The regulation is based on the NIH guidelines, with some important exceptions. In the Danish legislation there are no exempt organisms, ie, all work involving rDNA organisms must observe the guidelines and must be notified to the Danish Labour Inspection Service. As a part of the legislation, all activities involving rDNA organisms are being registered in a data base at the Danish National Institute of Occupational Health.

\section{REFERENCES}

1. Werner RG. Biobusiness in pharmaceutical industry. Arzneimittelforschung 1987;37:1086-93.

2. Primrose SB. The application of genetically engineered microorganisms in the production of drugs. J Appl Bacteriol 1986;61:99-116.

3. Münster O. Miljø og gensplejsning [Environment and genetic engineering]. Copenhagen: Ministry of the Environment, 1985.

4. Boyd HB, Broberg $\mathrm{O}$, Mikkelsen S. Risikohåndtering i bioteknologiske produktioner [Risk management in biotechnological productions]. Copenhagen: Technical University of Denmark, 1988. (Report from the Institute for Occupational Health.)

5. Wolpert L. DNA and its message. Lancet 1984;2:853-6. 
6. Ptashne M. How gene activators work. Sci Am 1989; 260:24-31.

7. Steitz JA. Snurps. Sci Am 1988;258:36-41.

8. Old RW, Primrose SB. Principles of gene manipulation. 3rd ed. Oxford: Blackwell Scientific Publications, 1985.

9. Dahl HH, Flavell RA, Grosveld FG. The use of genomic libraries for the isolation and study of eucaryotic genes. In: Williamson R, ed. Genetic engineering II. London: Academic Press, 1981:50-128.

10. Williams JG. The preparation and screening of a cDNA clone bank. In: Williamson R, ed. Genetic engineering I. London: Academic Press, 1981:2-61.

11. Novic RP. Plasmids. Sci Am 1980;243:76-92.

12. Rigby PWJ. Expression of cloned genes in eucaryotic cells using vector systems derived from viral replicons. In: Williamson R, ed. Genetic engineering III. London: Academic Press, 1982:83-134.

13. Giddins MD, Dabbah R, Grady LT, Rhodes CT. Scientific and regulatory aspects of macromolecular drugs and devices. Drug Dev Ind Pharm 1987;13:873-968.

14. Mizrahi A. Biologicals produced from animal cells in culture an overview. Biotechnol Adv 1988;6:207-20.

15. Tyo MA, Spier RE. Dense cultures of animal cells at the industrial scale. Enzyme Microb Technol 1987;9: 514-20.

16. Yamada H, Shimiza S. Microbial and enzymatic processes for the production of biologically and chemically useful compounds. Angew Chem Int Ed Engl 1988;27:622-42.

17. Griffin M, Magor AM. Possible use of microorganisms in the manufacture of plastics and synthetic fibers. Biotechnol Genet Eng Rev 1986;4:263-90.

18. Nicolaidis AA. Microbial mineral processing: the opportunities for genetic manipulation. J Chem Technol Biotechnol 1987;38:167-85.

19. Ganesan AT, Hoch JA. Bacillus molecular genetics and biotechnology applications. London: Academic Press, 1986.

20. Bisson LF. Strategies for improvement of industrial strains of Saccharomyces cerevisiae. World Biotechnol Rep 1986;2:103-16.

21. Poon CPC. Studies of the instantaneous death of airborne Escherichia coli. Am J Epidemiol 1966;84:1-9.

22. Cox CS. The cause of loss of vitality of airborne Escherichia coli K12. J Gen Microbiol 1969;57:77-80.

23. Durham OC. Airborne fungus spores as allergens. In: Moulton RF, ed. Aerobiology. Washington, DC: American Association for the Advancement of Science, 1942: $32-47$.

24. Smith LP, Huge-Jones ME. The weather factor in food and mouth disease epidemics. Nature 1969;223:712.

25. Organization for Economic Cooperation and Development. Recombinant DNA safety considerations. Paris: Organization for Economic Cooperation and Development, 1986.

26. Molin S, Klemm P, Poulsen LK, Biehl H, Gerdes K, Andersson P. Conditional suicide system for contain- ment of bacteria and plasmids. Bio/Technology 1987; $5: 1315-8$

27. Bej AK, Perlin MH, Atlas RM. Model suicide vector for containment of genetically engineered microorganisms. Appl Environ Microbiol 1988;54:2472-7.

28. Gill DM, Donowitz M. The risks involved in cloning toxin genes. In: Hardegree M, Tu AT, ed. Bacterial toxins. New York, NY: Marcel Dekker, 1988:453-65.

29. Sonea S. The global organism. Science 1988;241:38-45.

30. Liberman DF. Occupational hazards: illness in the microbiology laboratory. Publ Health Lab 1979;37: $118-29$.

31. Bouma JE, Lenski RA. Evolution of a bacteria/plasmid association. Nature 1988;335:351-2.

32. Küenzi M, Assi F, Chmiel A, et al. Safe biotechnology general considerations. Appl Microbiol Biotechnol 1985; $21: 1-6$.

33. Hardegree MC, Tu AT. Bacterial toxins. New York, NY: Marcel Dekker, 1988.

34. Spier R. Animal cells in culture: moving into the exponential phase. Trends Biotechnol 1988;6:2-6.

35. Epstein N, Epstein M. The hybridoma technology: $\mathbf{I}$. production of monoclonal antibodies. In: Mizrahi A, Liss A, ed. Adv Biotechnol Processes 1986:6:179-218.

36. Hooper ML. Mammalian cell genetics. New York, NY: John Wiley, 1985.

37. Levine AS. Cancer cells: I. the transformed phenotype. Cold Spring Harbor, NY: Cold Spring Harbor Laboratories, 1984.

38. Bishop JM. Oncogenes. Sci Am 1982;246:68-80.

39. Hunter $T$. The proteins of oncogenes. Sci Am 1984;257:60-70.

40. Mufson RA, Gesner T. Lack of tumorigenicity of cellular DNA and oncogene DNA in newborn hamsters. In Vitro Momography 1985;6:168.

41. Palmiter RD, Norstedt G, Gelinas RE, Hammer RE. Metallothionein - human GH fusion genes stimulate growth of mice. Science 1983;222:809-14.

42. Van Brunt $\mathbf{J}$. Molecular farming: transgenic animals as bioreactors. Bio/Technology 1988;6:1149-54.

43. Chatigny M. Sampling airborne microorganisms. In: Lioy PJL, Lioy MJY, ed. Air sampling instruments. Cincinnati, $\mathrm{OH}$ : Governmental Industrial Hygienists, 1983: E2-E9.

44. Saiki RK, Scharf S, Faloona F, et al. Enzymatic amplification of Beta-globin genomic sequences and restriction site analysis for diagnosis of sickle cell anemia. Science $1985 ; 230: 1350-4$.

45. Holden C. Looking at genes in the workplace. Science 1982;217:336-7.

46. President's Commission for the Study of Ethical Problems in Medicine Biomedicine and Behavioral Research Report. Splicing life; a report on the social and ethical issues of genetic engineering with human beings. Washington, DC: Government Printing Office, 1982.

Received for publication: 17 July 1989 